\title{
Emergency Echocardiography, Second Edition
}

\author{
Aleksandar N. Neskovic, Frank A. Flachskampf, Michael H. Picard. 252 pages, 2016. CRC \\ Press, Taylor \& Francis Group. ISBN 9781482243857
}

\author{
Vincent Lecluyse, MD
}

Received: 25 December 2016/Revised: 29 December 2016/Accepted: 23 January 2017/Published online: 31 January 2017

(C) Canadian Anesthesiologists' Society 2017

The vast potential for echocardiography to enhance perioperative patient assessment is well recognized. Indeed, most anesthesiologists enthusiastically welcome the opportunity to review a complete echocardiography report before they participate in the care of any sick patient in an emergency setting. With this growing interest and clinical experience, it is not surprising that research in the field of echocardiography is highly active, producing rapid expansion of textbook offerings.

Emergency Echocardiography is a recent addition to this growing echocardiography library. Written mostly by cardiologists, with some contributions from anesthesiologists, it appears very well designed, with 19 short chapters (10-15 pages each). The chapters cover mostly acute cardiac conditions-such as ischemia, cardiac failure, valvular disease, aortic dissection-with the addition of a few more esoteric chapters that address topics such as chest trauma, emergency department echocardiography, and pocket-sized ultrasound devices. The structures of the chapters are relatively similar. They begin with a description of a typical clinical scenario, move on to an overview of the differential diagnosis, followed by the echocardiographic presentation, and then an evaluation of the pathology in question. By ending each section with basic patient management considerations, the authors replicate the bedside cerebral process, making the read easy and intuitive.

The importance of visual aids in any book on ultrasound imaging cannot be understated, and Emergency Echocardiography delivers, with numerous clear, well-

V. Lecluyse, MD ( $\bowtie)$

Royal Melbourne Hospital, Melbourne, Australia

e-mail: vincent.lecluyse@gmail.com labelled, high-quality ultrasound snapshots. In addition, each chapter references multiple videos with a $\mathrm{QR}$ code and URL link. These 20- to 40-sec clips are easily accessible with any smartphone QR code scanner application and provide welcomed realism to the twodimensional still images found in the book. Unfortunately, the online E-book edition access code provided for the purpose of this review caused an error message to appear when the code was entered, making access impossible. One has to assume that the videos are directly accessible in the electronic version of the book. Another strength of this book is that it does not restrict itself to any specific image acquisition modality. Many other such books tend to focus on either transthoracic (TTE) or transesophageal (TEE) echocardiography, usually not both. Indeed, the focus in Emergency Echocardiography is clearly on the pathologies, pointing out the pros and cons (i.e., usefulness) of one or both echocardiography approaches. Thus, TTE and/or TEE are discussed only in the context of the medical scenario in question.

An important qualifier must be made from the start-the reader must be aware that this book is not meant to teach one how to perform an echocardiography examination but, rather, to illustrate multiple ways to integrate the examination into the larger picture of classic medical evaluation and management. As technical skills are not addressed in the book's curriculum and because advanced concepts of echocardiography are often discussed without having been extensively explained, this book is certainly not intended for novice or untrained echocardiographers. However, any physician with the skills and understanding necessary to surpass this initial knowledge gap would certainly be comfortable with the material subsequently covered. Although, arguably, this is a strength of the book, 
it does make it more difficult to understand the practitioner to whom this book would be most relevant, as it clearly requires a great deal of preexisting knowledge to be properly utilized. Yet, having this level of training in echocardiography might make the content somewhat redundant.

Another limitation of the book would be the expectation of acute, dramatic, life-saving scenarios suggested by the title itself: Emergency Echocardiography. Although very interesting on their own, the chapters regarding subjects such as native valve disease, prosthetic valves, cardiac tumours, cardiac stress testing, and ventricular assist devices make the book seem more like the conventional echocardiography that belongs in a cardiac surgery review manual rather than a book focussed on resuscitation complemented with echocardiography. In addition, current use of echocardiography in the emergency setting generally extends beyond only the evaluation of the heart. Therefore, the absence of any reference to abdominal or lung ultrasonography leaves the reader with an impression of missing content. Hemodynamic instability could originate from many causes other than heart disease. In addition, even when dealing with a purely cardiac disease, the severity of said disease can often be evaluated by its echocardiographic repercussion on other intrathoracic or abdominal organs. The book title, however, refers only to echocardiography. When faced with an unstable patient in an emergency situation, though, I find it difficult to justify limiting oneself uniquely to cardiac evaluation.

In summary, despite the interesting case-by-case presentations and high-quality visual media support, the book Emergency Echocardiography's potential usefulness is difficult to define because of the high level of prerequisite skills required to appreciate fully its content and its single organ approach, which makes it appear somewhat unidimensional. Therefore, this book would probably best cater to advanced ultrasonography practitioners looking to expand the clinical application of their skills. Beginners would probably do better to look to a different source for their first contact with echocardiography.

Conflicts of interest None declared.

Editorial responsibility This submission was handled by Dr. Hilary P. Grocott, Editor-in-Chief, Canadian Journal of Anesthesia. 